\title{
ON THE AGE OF GPS RADIO SOURCES
}

\author{
JOEL C. CARVALHO \\ Departamento de Fisica - UFRN - C.P. 1641 \\ 59072-970 Natal - RN - Brazil
}

The GHz-Peaked-Spectrum (GPS) radio sources are galaxies and quasars with compact structures on scales of tens to hundreds of parsecs and spectrum peaking around $1 \mathrm{GHz}$. Phillips and Mutel (1982) suggested that the double GPS sources (also called compact doubles, CD) are an early stage of the evolution of the classical double sources of Fanaroff-Riley class II type. We have presented a model (Carvalho, 1985) in which we show that the properties of the CD sources are consistent with such a scenario. Their ages would be of the order of $10^{4} \mathrm{yr}$.

Recent observations of diffuse extended radio emission around some (20\%) GPS sources, indicate that this sub-class may not be as young as initially thought. O'Dea et al. (1991) suggested that they would be smothered radio galaxies, a consequence of a recent increasing in the gas density near the center of the host galaxy due to an interaction or merger with a companion. Indeed, numerical simulations (De Young, 1990) show that jets can be drastically decelerated by dense clouds which are later destroyed by them. This would keep the source confined to the inner region of the galaxy. Thus, if the medium surrounding the radio source is very cloudy, the propagation will be slow and consequently the age will be larger than that deduced from a uniform medium (O'Dea et al., 1991). We have constructed a simplified model and analytically calculated the average velocity of the jet in a clumpy medium to found

$$
V=\left[1+R_{c} \lambda \frac{R_{c}}{R_{j}} \sqrt{\frac{n_{c}}{n_{j}}}\right]^{-1} V_{j} .
$$

Here $R$ is the radius, $n$ the particle number density, $\lambda$ is the number of clouds per unit length and the indices $c$ and $j$ refer to the cloud and jet respectively. The jet velocity in a homogeneous medium is $V_{j}$.

For characteristic values of the quantities we find $V \sim 10^{-2}-10^{-1} V_{j}$. We calculated the age of the GPS sources using this velocity and found it to be of the order of $10^{5}-10^{6} \mathrm{yr}$. This is near the range of ages of extended radio sources $\left(10^{6}-10^{7} \mathrm{yr}\right)$.

\section{References}

Carvalho, J.C. 1985, Mont. Not. R. Astron. Soc.215 463.

De Young, D.S. 1990, Astrophys. J.371 69.

O'Dea, C.P., Baum, S.A. and Stanghellini, C. 1991, Astrophys. J.380 66.

Phillips, R.B. and Mutel, R.L. 1982, Astron. Astrophys.106 21.

424

T. J.-L. Courvoisier and A. Blecha: Multi-Wavelength Continuum Emission of AGN, 424.

(C) 1994 IAU. Printed in the Netherlands. 